Revista Brasileira de Agricultura Irrigada v.13, nº.5, p. 3676 - 3682, 2019

ISSN 1982-7679 (On-line)

Fortaleza, CE, INOVAGRI - http://www.inovagri.org.br

DOI: $10.7127 /$ rbai.v13n5001130

Protocolo 1130.19 - 27/03/2020

Aprovado em 04/04/2020

\title{
DETECÇÃO DA EXPANSÃO DO CULTIVO DE BANANA NO CARIRI CEARENSE POR IMAGENS ORBITAIS
}

Carlos Wagner Oliveira ${ }^{1}$, Willian Nunes da Silva², Paulo Eduardo Rolim Campos ${ }^{3}$, Ana Célia Maia Meireles $^{4}$, Daliane da Silva Batista ${ }^{5}$

\section{RESUMO}

Este trabalho tem como objetivo identificar a expansão do cultivo de Banana (Musa $s p)$ nos municípios de Barbalha e Missão Velha, tomando por base um espaço temporal de 8 anos, usando para isso imagens de 2010 (Landsat 5) e 2018 (Landsat 8), adquiridas pelo site da USGS (United States Geological Survey). Para tal, fez-se o uso do software Qgis na versão 2.18 para o ajustes das imagens adquiridas, cômputo dos valores de NDVI (Normalized difference vegetation index) e, através do sensoriamento remoto, efetuou-se a detecção e a comparação de quais áreas de vegetação nativa ocorreram mudanças significativas no polo irrigado de produção de banana, localizados na região do Cariri cearense. Os resultados mostram que ocorreu uma mudança de $10 \%$ da vegetação na comparação entre os anos, um incremento de área de 1052 ha. Portanto foi concluído que com as técnicas de sensoriamento remoto e de geoprocessamento foi possível detectar mudanças espectrais na superfície da vegetação e avaliar o tamanho das alterações ocorridas durante o período no qual foi estudado, em que será de suma importância para o desenvolvimento agrícola sustentável na região.

Palavras-Chave: sensoriamento remoto, ndvi, landsat, musa.

\section{DETECTION OF BANANA CROP EXPANSION IN CARIRI CEARENSE BY ORBITAL IMAGES}

\begin{abstract}
The objective of this paper is to indicate the expansion of Banana (Musa sp) cultivation in the municipalities of Barbalha and Missão Velha, based on an 8 - year temporal space, using images from 2010 (Landsat 5) and 2018 (Landsat 8), acquired by the USGS (United States Geological Survey) website. For this purpose, the Qgis software version 2.18 was used to adjust the acquired

\footnotetext{
${ }^{1} \mathrm{PhD}$ em Biosystems Engineering, Professor da UFCA, Crato, Ceará, Brasil e-mail: carlos.oliveira@ufca.edu.br

${ }^{2}$ Graduando em Agronomia, UFCA, Crato, Ceará, Brasil. e-mail: willyannunes72@gmail.com

3 Doutorando em Agroecologia, UNEB, Universidade Estado da Bahia, Juazeiro, Bahia, Brasil. e-mail: permaculturakariry@gmail.com

${ }^{4}$ Doutora em Engenharia Civil, Professora da UFCA, Crato, Ceará, Brasil. e-mail: ana.meireles@ufca.edu.br

${ }^{5}$ Graduanda em Agronomia, UFCA, Crato, Ceará, Brasil. e-mail: dalianeagrimessura@gmail.com
} 
images, to compute the values of NDVI (Normalized difference vegetation index) and, through remote sensing, it was possible to detect and compare which areas of native vegetation occurred significant changes in the irrigated pole of banana production, located in the Cariri region of Ceará. The results show that $10 \%$ change in the vegetation occurred in the comparison between the years, equivalent an area of 1052 ha. It was concluded that with the remote sensing and geoprocessing techniques it was possible to detect spectral changes in the vegetation surface and to evaluate the size of the changes occurred during the period in which it was studied, which will be of great importance for sustainable agricultural development in the region.

Keywords: Remote sensing, ndvi, landsat, musa.

\section{INTRODUÇÃO}

Na Região Metropolitana Cariri, os municípios de Barbalha e Missão Velha são os maiores produtores de banana do polo agrícola irrigado da Região (ADECE, 2016). Com uso de irrigação e a mudança do cultivo da cana de açúcar para da banana houve um acréscimo de área e maior pressão na demanda por água. $\mathrm{O}$ cultivo de banana irrigada ocupa áreas de tamanhos relativamente expressivos, que promovem uma pressão sobre as fontes hídricas locais, pois a bananeira demanda consumo de água elevado, devido às suas características morfológicas (CARR, 2009). O entendimento de como a expansão, no tempo e no espaço, da área cultivada com banana provoca uma pressão sobre as fontes hídricas é de grande importância, uma vez que as cidades de Crato, Juazeiro do Norte, Barbalha e Missão Velha, localizados na RMC, são abastecidas em quase sua totalidade por água subterrânea. Além disso, o crescimento da população, da indústria e do setor agropecuário tem concorrido para um aumento no consumo da água, ocasionando depleção do aquífero (SANTOS et al., 2015). Dentre as variáveis utilizadas para avaliação da mudança do uso do solo, os índices de vegetação obtidos por sensoriamento remoto têm sido mais utilizados. Os índices de vegetação possibilitam a caracterização e quantificação de parâmetros biofísicos de florestas, culturas agronômicas e mudanças provocadas no uso do solo, pois reduzem a dimensão das informações multiespectrais fornecidas pelos satélites (GUILHERME et al., 2016). O índice de vegetação da diferença normalizada (do inglês Normalized Difference Vegetation Index - NDVI) é apontado como um dos mais aceitos índices para analisar a cobertura vegetal utilizando o sensoriamento remoto (HOLBEN et al., 1980)

Diante do exposto, esses métodos foram aplicados na região produtora de banana, localizada entre a cidade de Barbalha e Missão Velha, no cariri cearense, para analisar as alterações que ocorreram na área em um período de 8 anos.

\section{MATERIAL E MÉTODOS}

A área de estudo refere-se ao polo irrigado produtor de Banana (Musa sp), localizado na divisa entre os municípios de Barbalha e Missão Velha, no estado do Ceará, com as seguintes coordenadas geográficas: $7^{\circ} 19^{\prime}$ de latitude Sul, 39 $9^{\circ} 11^{\prime}$ de longitude Oeste e elevação de 403 metros em relação ao nível do mar, conforme Figura 1. Segundo classificação de Köppen-Geiger, o clima na localidade em estudo, é do tipo quente e úmido (Aw), com regime de chuvas tropical austral (w) dividido em duas etapas, sendo a primeira com chuvas concentradas no período de janeiro a abril, e a segunda mais seca iniciando em maio e perdurando até dezembro, totalizando $1.056 \mathrm{~mm}$ e $1.001 \mathrm{de}$ precipitação anual, respectivamente (LIMA et al., 2017). 


\section{DETECÇÃO DA EXPANSÃO DO CULTIVO DE BANANA NO CARIRI CEARENSE POR IMAGENS ORBITAIS}

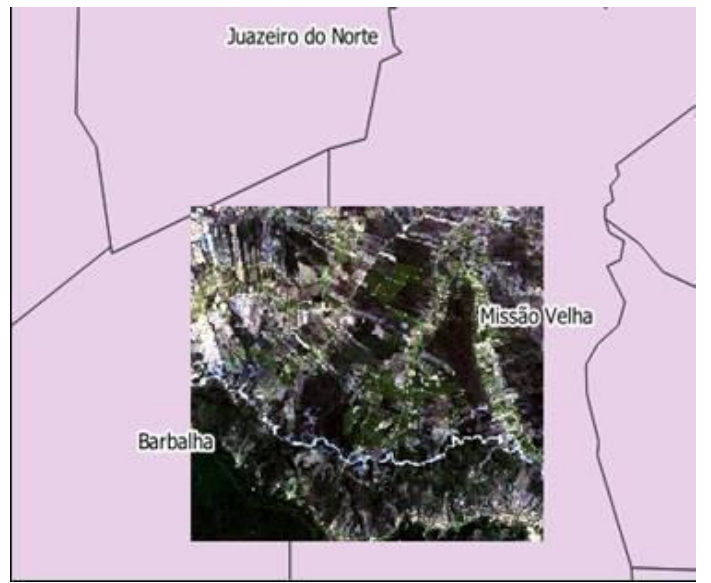

Figura 1. Localização da área do polo irrigado, com recorte da imagem em R3G2B1.

Neste estudo, foram utilizadas imagens do satélite Landsat 5 para o ano de 2010 (Figura 1) e do Landsat 8 para o ano de 2018, ambos na órbita 217 , linha 65 , captadas nos dias 13/10/2010 e 15/07/2018, obtidas através do site da USGS (United States Geological Survey) na plataforma "Earth Explorer". As imagens foram processadas através do software Qgis, na versão 2.18, ferramenta semi-automatic plugin (qgis.org).

O NDVI foi utilizado para determinar a densidade de fitomassa foliar fotossinteticamente ativa por unidade de área, uma vez que permite mostrar que quanto maior este índice de vegetação, mais densa é a fitomassa verde (MELO et al. 2011). A partir das imagens multiespectrais de satélite podese estimar esse índice, usando-se para isso as bandas do infravermelho próximo (760 a 900 nm) e do vermelho (630 a $690 \mathrm{~nm})$.

$\mathrm{NDVI}=(\boldsymbol{\rho i v}-\boldsymbol{\rho} \mathrm{v}) /(\boldsymbol{\rho i v}+\boldsymbol{\rho} \mathrm{v})$

Em que: NDVI - é o índice de vegetação da diferença normalizada; $\rho$ iv- reflectância infravermelho próximo; $\boldsymbol{\rho} \mathrm{v}$ - reflectância na faixa do vermelho. $\mathrm{Na}$ equação, considera-se apenas duas bandas do landsat 5 e 8 . No landsat 5 utiliza-se as bandas 3 (vermelho) e a banda 4 (Infravermelho), enquanto no landsat 8 foram utilizadas as bandas 4 (vermelho) e 5 (infravermelho).

A reflectância de cada banda ( $\boldsymbol{\rho})$ é definida como sendo a razão entre o fluxo de radiação solar refletido pela superfície e o fluxo de radiação solar global incidente, que é obtida através da equação (CHANDER et al., 2009):

$\boldsymbol{\rho}=\pi \mathrm{L} / \mathrm{K} \cos \mathrm{Zdr}$

Em que: L é a radiância espectral de cada banda, K é a irradiância solar espectral de cada banda no topo da atmosfera, $\mathrm{Z}$ é o ângulo zenital solar e dr é o quadrado da razão entre a distância média Terra-Sol em dado dia do ano.

A radiância monocromática $(\mathrm{L})$ de cada uma das bandas é obtida segundo a expressão:

$\mathrm{L}=\mathrm{G}(\mathrm{ND})+\mathrm{B}$

Em que: $\mathrm{G}$ é o ganho, ND é o número digital da imagem, e B é o intercepto.

Para equação 3, quando na descrição do produto (imagem) não estão claramente explícitos os valores de "G" e "B", adota-se os valores propostos por Chander et al. (2009).

\section{RESULTADOS E DISCUSSÃO}

Para o período estudado, nota-se que houve um aumento considerável do polo irrigado do ano de 2010 (Figura 2 e 3) com a diminuição da vegetação rasteira nativa comparada com a condição atual de 2018 (Figura 4 e 5). Essa mudança é acompanhada da abertura de novos poços para o uso na irrigação na produção de banana, aumentando assim a pressão sobre as reservas hídricas da 
Oliveira et al.

região. Vale salientar que, o déficit hídrico e a baixa pluviosidade na região nesta última década prejudicaram as recargas do aquífero, agravando a situação na região. Nessa perspectiva de variação da cobertura vegetal, na qual a cada ano ocorre um aumento na produção e a intensificação da atividade agrícola, acabam por gerar alterações ambientais, principalmente na área e no entorno do polo irrigado, no qual se concentram grandes áreas de empresas de comercialização de bananas no Nordeste.

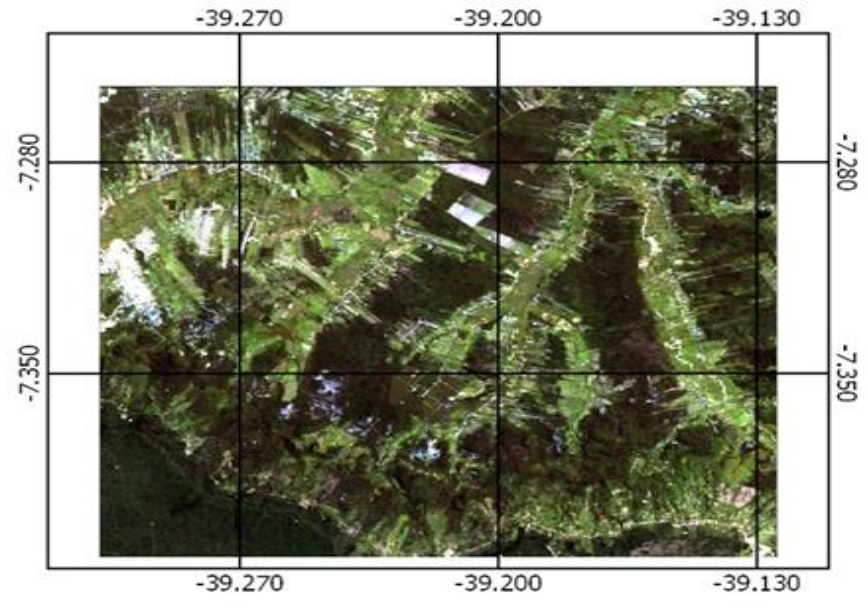

Figura 2. Imagem multiespectral R3G2B1, entorno Barbalha, Missão Velha, 2010.

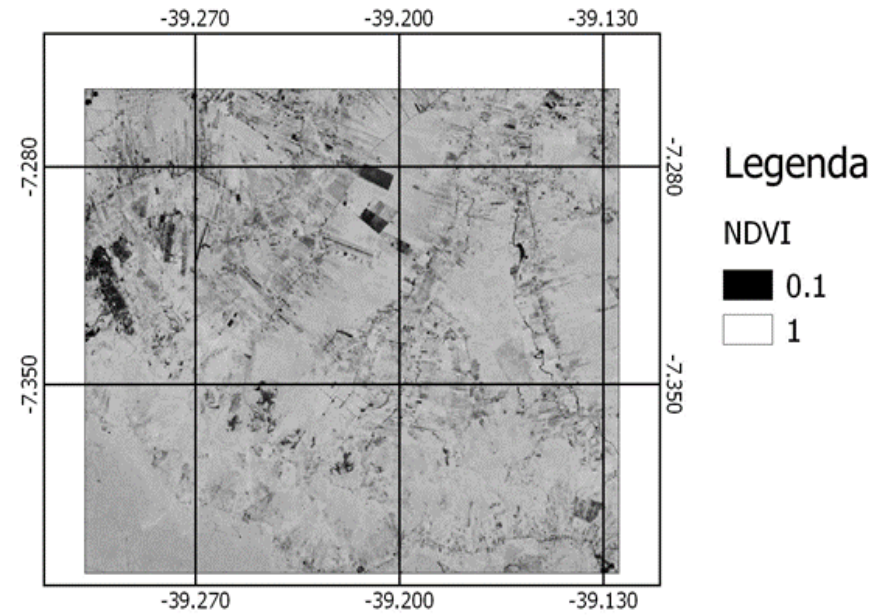

Figura 3. NDVI da área entorno Barbalha, Missão Velha, 2010.

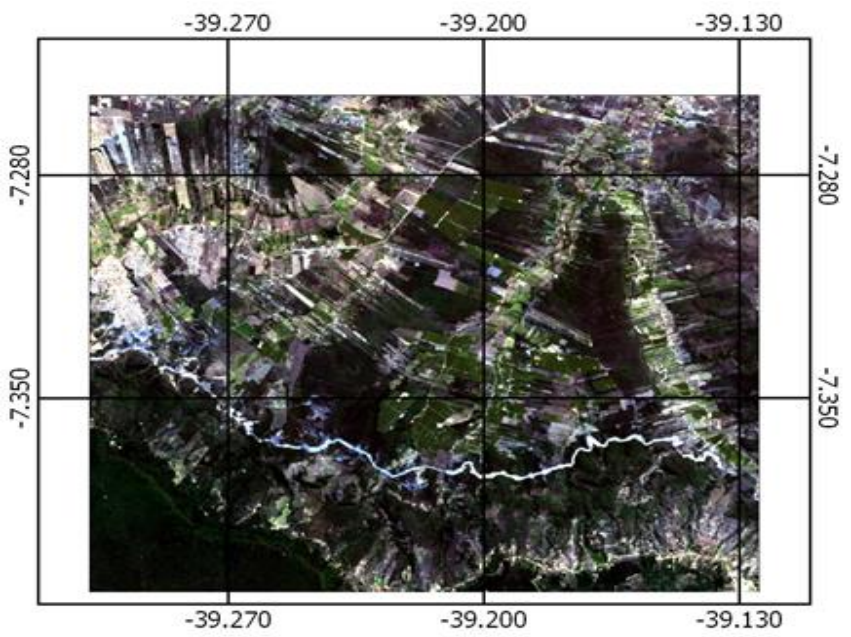

Figura 4. Imagem multiespectral R4G3B2, no entorno Barbalha, Missão Velha, 2018. 


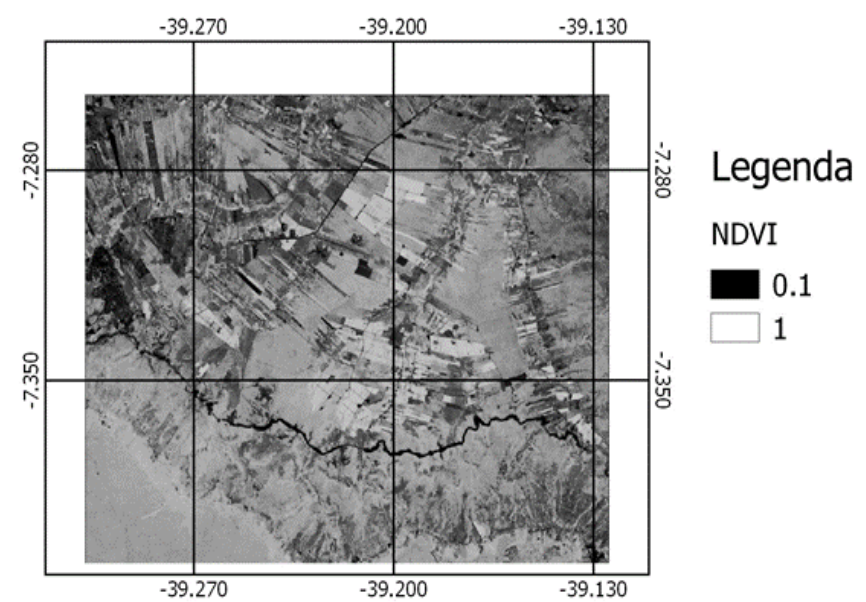

Figura 5. NDVI da área no entorno Barbalha, Missão Velha, 2018.

Nas figuras 6 e 7 pode-se observar melhor quais as áreas que mais se alteraram. $\mathrm{Na}$ cor vermelha áreas que tiveram redução no valor do NDVI em $10 \%$ e nas áreas na cor preta, as que tiveram um número quase idêntico, com pouquíssimas alterações.

Já as áreas onde houve incremento de cultivo de banana irrigada, os valores de NDVI demonstraram um acréscimo de pelo menos $10 \%$, observados na figura 7 pela coloração verde. Este valor corresponde a um aumento de 1.052 hectares.

Na Figura 7 é de se ressaltar grandes áreas onde houve redução no valor do NDVI, para 2018, mostradas em vermelho (decremento mínimo de 10\%). Mesmo no mês de julho, próximo da estação chuvosa, esses baixos valores de NDVI, mostram a susceptibilidade da área com solos expostos e baixa cobertura vegetal.

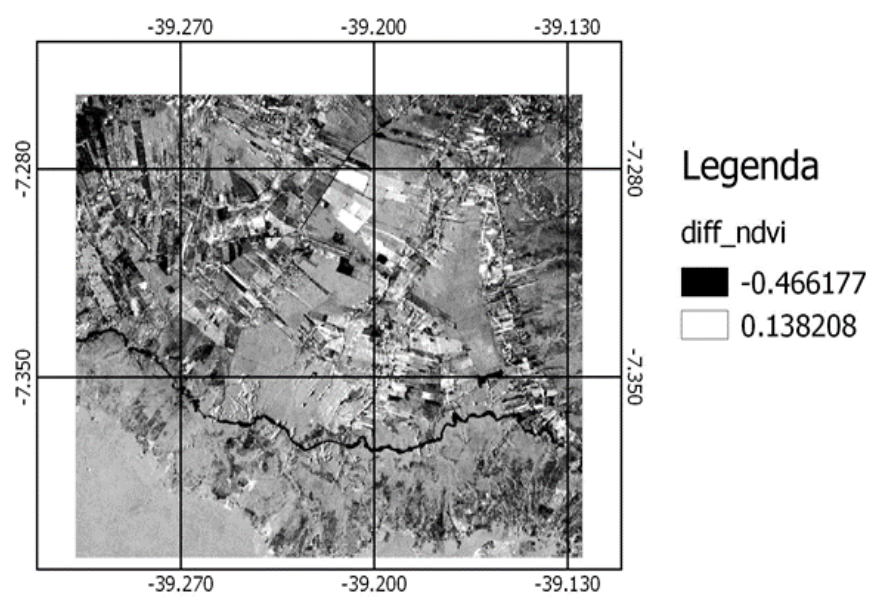

Figura 6. Diferença entre os valores de NDVI entre 2010 e 2018. 


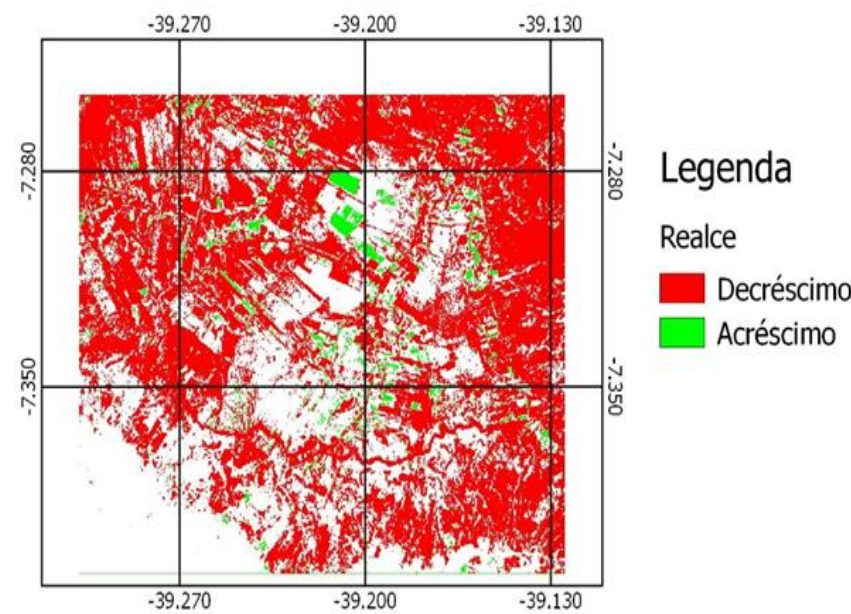

Figura 7. Realce áreas que tiveram incremento (verde) ou decremento (vermelho) do NDVI em pelo menos $10 \%$ para o período entre 2010 e 2018.

Essa tendência de aumento das áreas irrigadas em polos do Nordeste Brasileiro, também foi observada por Lira et al.(2011), usando imagens de satélite Landsat 5 e cálculo do NDVI, no Sub-Médio São Francisco, que durante período de estudo houve aumento área de cana-de açúcar. Estudos semelhantes, como o uso NDVI na microbacia do riacho dos cavalos em Crateús-CE (MELO et al., 2011), avaliando o estado geral de degradação ambiental, mostram claramente uma considerável variação da cobertura vegetal e, sem dúvida, dos padrões de uso da terra entre diferentes anos. Em 1979, o setor que corresponde ao índice de cobertura vegetal era de aproximadamente $22 \mathrm{~km}^{2}$, ou $0,5 \%$ da área da microbacia, já no ano de 2006 essa área expandiu para $108 \mathrm{~km}^{2}$, denotando assim a degradação ocorrida na região.

Dentro do nosso recorte de estudo, considerando as áreas contendo cultivo irrigado, esta área foi estimada em aproximadamente 1.900 hectares. Na Tabela 1 abaixo, observa-se os valores resultantes do cálculo das áreas empresariais irrigadas em comparação com dados da COGERH e IBGE. Entende-se que os dados obtidos na pesquisa através das imagens de satélite são os mais atuais, já que a imagem é de outubro de 2018, sendo os dados da COGERH referentes ao ano de 2015. As informações oriundas do IBGE são de 2016. Entretanto estas ultimas, não fazem distinção entre as culturas irrigadas e as não irrigadas.
Tabela 1. Áreas (ha) empresariais irrigadas em Barbalha e Missão Velha.

\begin{tabular}{ccc}
\hline $\begin{array}{c}\text { COGERH } \\
(2015)\end{array}$ & $\begin{array}{c}\text { IBGE } \\
(2016)\end{array}$ & $\begin{array}{c}\text { Obtido } \\
(2018)\end{array}$ \\
\hline 1798 & 1950 & 1900 \\
\hline
\end{tabular}

O valor obtido na pesquisa se aproxima com os dados da COGERH e IBGE, o que confere determinada confiança na metodologia usada.

\section{CONCLUSÕES}

No período observado, ocorreu uma mudança da vegetação nativa característica da região, somada com a drástica mudança no ciclo hidrológico e pela rápida expansão da área cultivada, que a produção de banana teve na região na região ao longo dos últimos anos, o que foi confirmado pelo NDVI. No qual a área próxima do irrigado sofreu uma mudança de pelo menos $10 \%$ no valor do NDVI, esse incremento representa 1.052 hectares, adicionados a produção irrigada na área estudada. Neste trabalho, a área irrigada estimada por imagem está em torno de 1.900 hectares, o que corrobora com dados do IBGE.

\section{REFERÊNCIAS BIBLIOGRÁFICAS}

\author{
ADECE-AGÊNCIA DE \\ DESENVOLVIMENTO DO ESTADO DO \\ CEARÁ S/A. Perfil da Produção de Frutas
}




\section{DETECÇÃO DA EXPANSÃO DO CULTIVO DE BANANA NO CARIRI CEARENSE POR IMAGENS ORBITAIS}

Brasil e Ceará. In: FRUTAL, 20,, 2013, Fortaleza. Palestra.. Fortaleza: FRUTAL, 2013. Disponível em: <http://www.adece.ce.gov.br/phocadownload/ Agronegocio/perfil_da_producão $>$. Acesso em: 26 nov. 2016.

CARR, M.K.V., 2009. The water relations and irrigation requirements of banana (musa spp.). Experimental Agriculture. 45, 333. https://doi.org/10.1017/S001447970900787X

CHANDER, G., MARKHAM, B.L., HELDER, D.L. 2009. Summary of current radiometric calibration coefficients for Landsat MSS, TM, ETM+, and EO-1 ALI sensors. Remote Sensing of Environment, 113, 893-903.

COGERH. 2017. Estudos das Águas Subterrâneas da Bacia do Araripe (Relatório Síntese dos Estudos Qualitativos e Quantitativos). COGERH/IPECE/SRH, Fortaleza.

GUILHERME, A.P., MOTA, A.B. DOS S., MOTA, D. DOS S., MACHADO, N.G., BIUDES, M.S. 2016.Uso de índice de vegetação para caracterizar a mudança no uso do solo em Coari-AM. Sociedade \& Natureza, 28, 301-310.

HOLBEN, B. N.; TUCKER, C. J.; FAN, C. J. 1980.Spectral assessment of soybean leaf area and leaf biomass. Photogrammetric Engineering and Remote Sensing, v.46, pp.651-656.
IBGE. Produção Agrícola - Lavoura Permanente / Missão Velha (CE). Instituto Brasileiro de Geografia e Estatística. Disponível em: $<$ https://cidades.ibge.gov.br/brasil/ce/missaovelha/pesquisa/15/11863?ano=2012> Acesso em: 10/12/2018

LIM, M.T.V., MEIRELES, A.C.M., OLIVEIRA, C.W., NASCIMENTO, M.T.B., 2017. Koppen-Geiger and Thornthwaite climatic classification for the metropolitan region of the Cariri, Ceará. Revista GEAMA, 3, 136-143.

LIRA, V.M., SILVA, B. B, NETO, J.D., AZEVEDO, C.A.V., FRANCO, E.S., 2011. Variação sazonal da cobertura vegetal em áreas do submédio são francisco a partir de dados ndvi e imagens tm-landsat 5 . Enciclopédia Biosfera 7, 1-9.

MELO, E. T., SALES, M. C. L., OLIVEIRA, J. G. B. 2011.Aplicação do Índice de Vegetação por Diferença Normalizada (NDVI) para análise da degradação ambiental da microbacia hidrográfica do Riacho dos Cavalos, Crateús-CE. O Espaço Geográfico em Análise, 23, p. 520-533.

SANTOS, M.A.P., SANTOS, V.S., FRANCA, R.M., CHAVES, M.S., 2015. Águas subterrâneas: Um estudo acerca da superexplotação sobre a microbacia III do Rio Salgado. Revista Verde de Agroecologia e Desenvolvimento Sustentável 10, 11. https://doi.org/10.18378/rvads.v10i4.3538 